Produto \& Produção, vol. 11, n. 1, p. 113 - 123, fev. 2010

Edição Metrologia

\title{
Validação de Metodologia Analítica para a Determinação de Hidrocarbonetos Policíclicos Aromáticos em Solução
}

\author{
Evelyn de F. Guimarães, MSc. ${ }^{1},{ }^{3}$ \\ efguimaraes@inmetro.gov.br \\ Eliane Cristina Pires do Rego, MSc..$^{1}{ }^{2}$ \\ ecrego@inmetro.gov.br \\ Helen Cristine Moreira Cunha ${ }^{1}{ }^{2}$ \\ helenzinhamoreira@hotmail.com \\ Janaína M. Rodrigues, $\mathrm{Dr}^{1}{ }^{2}$ \\ imrodrigues@inmetro.gov.br \\ José Daniel Figueroa Villar, $\mathrm{Dr}^{3}$ \\ figueroa@ime.eb.br \\ Valnei Smarçaro da Cunha, $\mathrm{Dr}^{1}$ \\ vscunha@inmetro.gov.br
}

\begin{abstract}
RESUMO
Este trabalho apresenta a validação da metodologia analítica para determinação de dezesseis hidrocarbonetos policíclicos aromáticos em solução. A técnica de CG/EM combinada com o princípio de diluição isotópica foi usada para melhor exatidão e precisão dos resultados. Foram determinados parâmetros de desempenho do método e os resultados encontrados foram satisfatórios.
\end{abstract}

Palavras-chave: Hidrocarbonetos Policíclicos Aromáticos (HPAs), Validação, Material de Referência Certificado (MRC).

\footnotetext{
${ }^{1}$ Divisão de Metrologia Química, Diretoria de Metrologia Científica e Industrial, Instituto Nacional de Metrologia, Normalização e Qualidade Industrial - INMETRO, Duque de Caxias, Brasil.

${ }^{2}$ Laboratório de Análise Orgânica - INMETRO, Duque de Caxias, Brasil.

${ }^{3}$ Departamento de Química, Instituto Militar de Engenharia - IME, Rio de Janeiro, Brasil.
} 


\section{INTRODUÇÃO}

Hidrocarbonetos Policíclicos Aromáticos (HPAs) são substâncias orgânicas de persistência ambiental e potencial tóxico (carcinogênicos e/ou mutagênicos). A Figura 1 mostra a estrutura química de dezesseis HPAs considerados poluentes prioritários pela Agência de Proteção Ambiental dos Estados Unidos (U.S.EPA) (Mastral, 2000, p. 3051- 3057).

A qualidade das medições destes compostos é de grande interesse para a saúde pública e para os organismos regulatórios de vários países (IARC, 2007). Devido à importância da determinação confiável destes compostos, a precisão e a exatidão tornam-se fatores relevantes para o processo. Desta forma, a abordagem metrológica, compreendida pela Metrologia Química torna-se fundamental (De Bièvre, 1997, p. 67-75).

Para superar estes desafios, o Inmetro tem o importante papel de fornecer a rastreabilidade e a confiabilidade das medições e a Divisão de Metrologia Química (Dquim) tem a responsabilidade de desenvolver procedimentos de referência, implementar métodos primários, desenvolver e certificar materiais de referência.

$\mathrm{Na}$ determinação de contaminantes ambientais por institutos de metrologia, bem como por laboratórios de pesquisa, tem sido empregada a técnica de Espectrometria de Massas com Diluição Isotópica (EMDI), com o objetivo de prover maior exatidão às medições realizadas (Veyrand et al. 2007, p. 333-344; Itoh et al. 2006, p. 246-252; Planas et al. 2006, p. 220-230; Milton, 2001, p. 289-296). Esta técnica é considerada como um método primário de medição pelo Comitê Consultivo para a Quantidade de Matéria (CCQM), sendo rastreável ao Sistema Internacional de Unidades (SI) (Milton, 2001, p. 289-296; Quinn, 1997, p. 61-65).

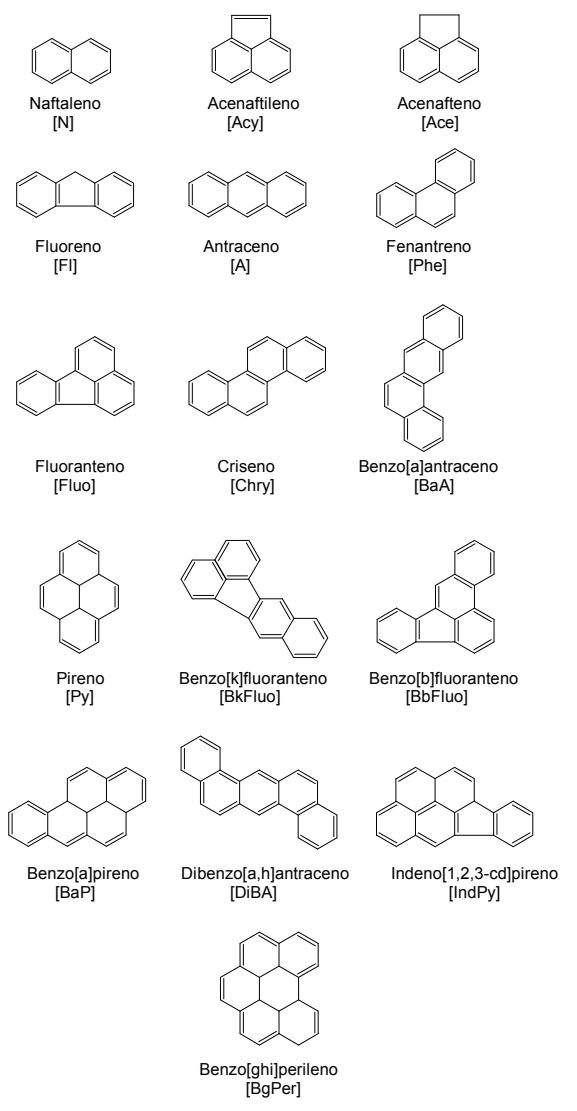


Figura 1 - Estrutura química dos dezesseis HPAs prioritários pela U.S.EPA e suas abreviações.

Neste estudo, foi desenvolvido e validado um método para determinação de dezesseis HPAs em solução através de cromatografia à gás com espectrometria de massas, utilizando a técnica de diluição isotópica (CG/EMDI).

\section{OBJETIVO}

O presente trabalho teve como objetivo a validação da metodologia analítica utilizada nos estudos da certificação de um candidato a material de referência para dezesseis HPAs em tolueno, através de CG/EMDI.

\section{MÉTODO}

A metodologia analítica empregando a técnica de CG/EMDI foi implementada e validada para a determinação de dezesseis HPAs em tolueno, utilizando os respectivos HPAs deuterados como padrões internos (PIs).

Inicialmente foram preparadas soluções estoque para os 16 HPAs nativos e os 16 HPAs deuterados. Estas soluções foram preparadas gravimetricamente em balança analítica a partir dos padrões individuais de cada HPA nativo e deuterado, utilizando tolueno grau pesticida como solvente. A partir destas soluções, preparouse gravimetricamente uma solução contendo $5 \mu \mathrm{g} / \mathrm{g}$ de cada composto em tolueno. Esta solução foi utilizada para a otimização do método em cromatógrafo a gás (CG) modelo CP-3800 acoplado ao espectrômetro de massas Ion Trap (EM-IT) Varian Saturn 2000, de modo a obter a resolução adequada entre os compostos, confirmada pela análise dos espectros de massas obtidos.

A análise foi realizada sob as seguintes condições: razão de splitless 1:10 após $1 \mathrm{~min}$; volume de injeção de $1 \mu \mathrm{L}$; temperaturas do injetor, do trap, da linha de transferência e do manifold foram ajustadas em $290^{\circ} \mathrm{C}, 230^{\circ} \mathrm{C}, 280^{\circ} \mathrm{C}$ e $80^{\circ} \mathrm{C}$, respectivamente; modo Scan e SIS (íon seletivo), coluna cromatográfica DB-5ms (60 $\mathrm{m} \times 250 \mathrm{~mm} \times 0,25 \mathrm{~m})$; programação do forno: $60^{\circ} \mathrm{C}(2 \mathrm{~min}), 120^{\circ} \mathrm{C}\left(20^{\circ} \mathrm{C} / \mathrm{min} ; 2\right.$ $\mathrm{min}), 180{ }^{\circ} \mathrm{C}\left(6,0{ }^{\circ} \mathrm{C} / \mathrm{min} ; 2 \mathrm{~min}\right), 290{ }^{\circ} \mathrm{C}\left(3,0{ }^{\circ} \mathrm{C} / \mathrm{min} ; 29,33 \mathrm{~min}\right)$, sendo o tempo total de análise de 85 minutos. Foi usado hélio 6.0 como gás de arraste com fluxo constante de 1,0 $\mathrm{mL} / \mathrm{min}$ (Figura 2). 


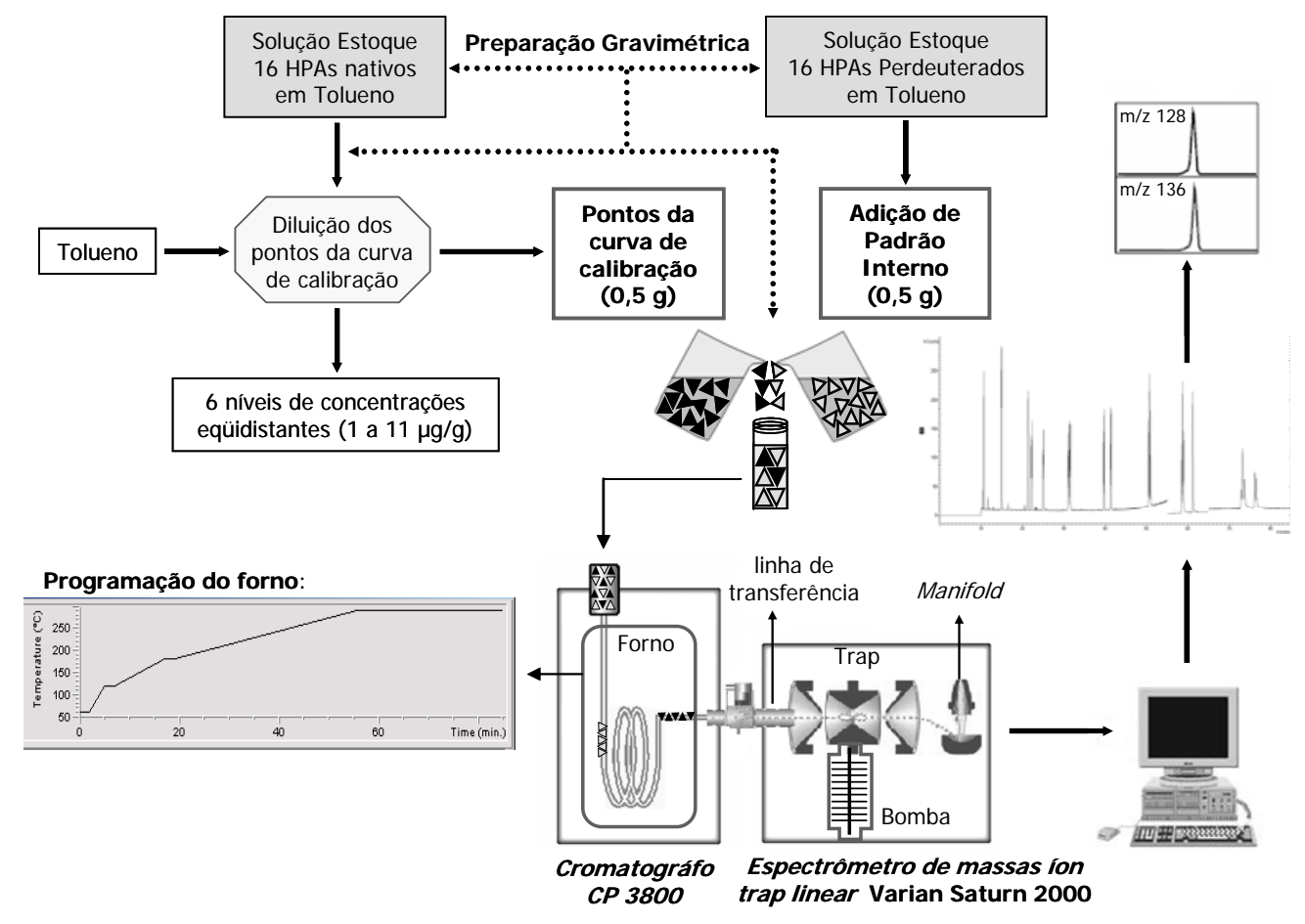

Figura 2 - Esquema de validação de metodologia analítica para a determinação de HPAs em solução.

A Tabela 1 apresenta os tempos de retenção obtidos para cada HPA e seu respectivo padrão interno $(\mathrm{PI})$, bem como os íons escolhidos para monitoramento e quantificação.

Tabela 1 - Tempo de retenção e m/z dos HPAs e seus Pls.

\begin{tabular}{|c|c|c|}
\hline HPAs nativo / deuterado & TR (min) & Íons $(\mathrm{m} / \mathrm{z})$ \\
\hline $\mathrm{N} /$ Naftaleno perdeuterado ${ }^{\mathrm{a}}$ & $15,11 / 15,05$ & $128 / 136$ \\
\hline Acy / Acenaftileno perdeuterado ${ }^{a}$ & $21,52 / 21,48$ & $152 / 160$ \\
\hline Ace / Acenafteno perdeuterado ${ }^{\mathrm{a}}$ & $22,42 / 22,25$ & $154 / 164$ \\
\hline $\mathrm{Fl} /$ Fluoreno perdeuterado ${ }^{\mathrm{a}}$ & $25,26 / 25,08$ & $166 / 176$ \\
\hline Phe / Fenantreno perdeuterado ${ }^{a}$ & $31,38 / 31,19$ & $178 / 188,3$ \\
\hline A / Antraceno perdeuterado ${ }^{a}$ & $31,73 / 31,57$ & $178 / 188,3$ \\
\hline Fluo / Fluoranteno perdeuterado ${ }^{a}$ & 39,89 / 39,76 & $202 / 212,3$ \\
\hline Py / Pireno perdeuterado ${ }^{\mathrm{a}}$ & $41,56 / 41,42$ & $202 / 212,3$ \\
\hline $\mathrm{BaA} / \mathrm{Benz}[\mathrm{a}]$ antraceno perdeuterado ${ }^{\mathrm{a}}$ & $50,77 / 50,59$ & 228,2 / 240,4 \\
\hline Chry / Criseno pereuterado ${ }^{\mathrm{a}}$ & $51,03 / 50,82$ & $228,2 / 240,4$ \\
\hline BbFluo / Benzo[b]fluoranteno perdeuterado ${ }^{\mathrm{a}}$ & $58,78 / 58,59$ & $252,4 / 264,4$ \\
\hline BkFluo / Benzo[k]fluoranteno perdeuterado ${ }^{\mathrm{a}}$ & $59,01 / 58,84$ & $252,4 / 264,4$ \\
\hline $\mathrm{BaP} / \mathrm{Benzo}[\mathrm{a}]$ pireno perdeuterado ${ }^{\mathrm{a}}$ & $61,47 / 61,25$ & $252,4 / 264,4$ \\
\hline IndPy / Indeno[1,2,3-cd]pireno perdeuterado ${ }^{\mathrm{a}}$ & $73,34 / 73,04$ & $276,4 / 288,4$ \\
\hline $\mathrm{DiBa} / \mathrm{Dibenzo}[a, h]$ antraceno perdeuterado ${ }^{\mathrm{a}}$ & $73,86 / 73,44$ & $278,4 / 292,4$ \\
\hline BgPer / Benzo[ghi]perileno perdeuterado ${ }^{\mathrm{a}}$ & $76,75 / 73,36$ & $276,4 / 288,4$ \\
\hline
\end{tabular}

(a) Padrão interno (PI)

Na preparação das curvas de calibração pelo método de padronização interna (diluição isotópica), as diluições também foram realizadas por gravimetria. Foram preparadas soluções-padrão das substâncias de interesse em seis níveis de concentrações eqüidistantes (1 a $11 \mu \mathrm{g} / \mathrm{g}$ ), às quais adicionou-se quantidade conhecida do padrão interno deuterado dos respectivos HPAs investigados. 
A validação do método desenvolvido foi realizada com base nos seguintes documentos: DOQ-CGCRE-008 do INMETRO, AOAC (Association of Analytical Communities) e ISO/IEC 17025 (INMETRO, 2007; AOAC, 1999; ISO/IEC 17025:2005, 2005).

Os parâmetros de desempenho avaliados foram: linearidade, exatidão, precisão, limite de detecção (LD) e limite de quantificação (LQ).

No estudo de linearidade foi avaliado se a faixa de trabalho usada apresentava-se linear para todos os HPAs estudados. Para isso, foram preparadas triplicadas em seis níveis de concentração $(1,3,5,7,9$ e $11 \mu \mathrm{g} / \mathrm{g})$. Cada curva de calibração foi construída através da relação entre área da substância / área do padrão interno e a razão de massa da substância / massa do padrão interno. Os valores de razão de área e razão de massa foram usados para obter a equação de regressão linear pelo método dos mínimos quadrados.

O coeficiente de correlação quadrado ( $r 2$ ) foi utilizado para avaliar a adequação da representação do modelo matemático expresso pela equação de reta. Um valor de r2 maior que 0,995 foi requerido para considerar o modelo linear. A variância ao longo da curva foi avaliada através do Teste de Cochran (ISO 5725, 1994).

Para avaliar a exatidão, foram realizados ensaios de recuperação de soluções controle dos HPAs abrangendo toda a faixa de trabalho $(2,6$ e $10 \mu \mathrm{g} / \mathrm{g})$, preparadas em triplicatas.

A exatidão também foi avaliada através do uso de material de referência certificado SRM 2260a fornecido pelo National Institute of Standards and Technology (SRM 2260a, 2004).

A precisão foi avaliada através de estudos de repetitividade, através das soluções controles utilizadas nos estudos de recuperação.

Os limites de detecção e quantificação (LD e LQ) foram determinados através do preparo de dez replicatas de uma solução em concentração inferior ao primeiro ponto da curva de calibração, sendo nível escolhido de $0,1 \mu \mathrm{g} / \mathrm{g}$.

\section{RESULTADOS}

Os valores encontrados para os parâmetros de desempenho determinados na validação da metodologia analítica para a determinação dos dezesseis HPAs em solução estão apresentados nas Tabelas 2, 3 e 4.

A Tabela 2 apresenta as informações referentes ao parâmetro Linearidade. A Tabela 3 mostra os resultados encontrados para os parâmetros Limite de Detecção (LD), Limite de Quantificação (LQ), Recuperação (Recup.) e Repetitividade (Repe). A Tabela 4 apresenta os resultados encontrados para o material de referência certificado (MRC) 2260a (NIST), avaliando o parâmetro exatidão. 
Tabela 2 - Parâmetro de validação: Linearidade

\begin{tabular}{|l|c|c|c|c|}
\hline \multicolumn{1}{|c|}{ HPAs } & $\begin{array}{c}\text { Lin. } \\
\mathrm{r}^{2}\end{array}$ & Equação de reta & Teste de Cochran & $\begin{array}{c}\text { Maior } \\
\text { DPR (\%) }\end{array}$ \\
\hline Naftaleno & 0,999 & $\mathrm{y}=1,128 \mathrm{x}-0,0601$ & HOMO & 0,73 \\
\hline Acenaftileno & 0,999 & $\mathrm{y}=1,140 \mathrm{x}-0,0969$ & HOMO & 1,65 \\
\hline Acenafteno & 0,999 & $\mathrm{y}=1,286 \mathrm{x}-0,1032$ & HETERO & 1,48 \\
\hline Fluoreno & 0,999 & $\mathrm{y}=1,517 \mathrm{x}-0,2151$ & HETERO & 1,01 \\
\hline Fenantreno & 0,999 & $\mathrm{y}=1,567 \mathrm{x}-0,1246$ & HETERO & 1,66 \\
\hline Antraceno & 0,998 & $\mathrm{y}=1,149 \mathrm{x}-0,1184$ & HOMO & 2,20 \\
\hline Fluoranteno & 0,999 & $\mathrm{y}=1,414 \mathrm{x}-0,0988$ & HOMO & 1,94 \\
\hline Pireno & 0,999 & $\mathrm{y}=1,603 \mathrm{x}-0,0953$ & HOMO & 1,62 \\
\hline Benzo(a)antraceno & 0,997 & $\mathrm{y}=1,204 \mathrm{x}-0,16$ & HOMO & 1,47 \\
\hline Criseno & 0,995 & $\mathrm{y}=0,980 \mathrm{x}-0,0499$ & HETERO & 5,41 \\
\hline Benzo(b)fluoranteno & 0,996 & $\mathrm{y}=1,581 \mathrm{x}-0,2436$ & HOMO & 2,28 \\
\hline Benzo(k)fluoranteno & 0,999 & $\mathrm{y}=1,236 \mathrm{x}-0,0519$ & HETERO & 5,54 \\
\hline Benzo(a)pireno & 0,999 & $\mathrm{y}=2,333 \mathrm{x}-0,2547$ & HOMO & 6,78 \\
\hline Indeno(1,2,3-cd)pireno & 0,995 & $\mathrm{y}=1,012 \mathrm{x}-0,159$ & HOMO & 2,35 \\
\hline Dibenzo(a,h)antraceno & 0,998 & $\mathrm{y}=1,404 \mathrm{x}-0,0402$ & HOMO & 6,25 \\
\hline Benzo(ghi)perileno & 0,995 & $\mathrm{y}=1,169 \mathrm{x}-0,0704$ & HETERO & 3,21 \\
\hline
\end{tabular}

Lin. = Linearidade; $\mathrm{DPR}=$ Desvio padrão relativo;

HOMO = Homocedástico; HETERO = Heterocedástico.

Tabela 3 - Parâmetros de validação: Limite de Detecção (LD), Limite de Quantificação (LQ), Recuperação (Recup.) e Repetitividade (Repe).

\begin{tabular}{|l|c|c|c|c|}
\hline \multicolumn{1}{|c|}{ HPAs } & $\begin{array}{c}\text { LD } \\
(\mu \mathrm{g} / \mathrm{g})\end{array}$ & $\begin{array}{c}\text { LQ } \\
(\mu \mathrm{g} / \mathrm{g})\end{array}$ & $\begin{array}{c}\text { Recup. } \\
(\%) \\
*\end{array}$ & $\begin{array}{c}\text { Repe (\%) } \\
(\mathrm{DPR}) \\
*\end{array}$ \\
\hline Naftaleno & 0,004 & 0,013 & $99-100$ & $0,42-0,82$ \\
\hline Acenaftileno & 0,007 & 0,023 & $98-100$ & $0,43-0,70$ \\
\hline Acenafteno & 0,009 & 0,032 & $98-101$ & $0,50-1,01$ \\
\hline Fluoreno & 0,017 & 0,057 & $100-102$ & $0,40-0,44$ \\
\hline Fenantreno & 0,015 & 0,049 & $99-103$ & $0,99-1,03$ \\
\hline Antraceno & 0,018 & 0,061 & $100-101$ & $1,00-1,02$ \\
\hline Fluoranteno & 0,009 & 0,030 & $99-100$ & $0,99-1,00$ \\
\hline Pireno & 0,015 & 0,050 & $98-100$ & $0,04-1,70$ \\
\hline Benzo(a)antraceno & 0,029 & 0,097 & $94-100$ & $0,71-1,79$ \\
\hline Criseno & 0,006 & 0,019 & $94-98$ & $0,27-1,56$ \\
\hline Benzo(b)fluoranteno & 0,032 & 0,106 & $95-100$ & $0,55-1,91$ \\
\hline Benzo(k)fluoranteno & 0,051 & 0,171 & $98-103$ & $0,36-0,68$ \\
\hline Benzo(a)pireno & 0,029 & 0,097 & $97-101$ & $1,68-4,57$ \\
\hline Indeno(1,2,3-cd)pireno & 0,013 & 0,043 & $96-101$ & $0,43-1,50$ \\
\hline Dibenzo(a,h)antraceno & 0,017 & 0,056 & $95-101$ & $0,28-1,48$ \\
\hline Benzo(ghi)perileno & 0,019 & 0,062 & $97-100$ & $0,38-2,08$ \\
\hline
\end{tabular}

DPR = Desvio padrão relativo;

* Valor mínimo e valor máximo encontrados. 
Tabela 4 - Parâmetro exatidão através do MRC 2260ª

\begin{tabular}{|l|c|c|c|c|c|}
\hline \multicolumn{1}{|c|}{ HPAs } & $\begin{array}{c}\text { Valor certificado } \\
(\mu \mathrm{g} / \mathrm{g})\end{array}$ & $\mathrm{U}$ & Valor obtido( $\mu \mathrm{g} / \mathrm{g})$ & ER (\%) & Recup. (\%) \\
\hline Naftaleno & 11,43 & 0,30 & 11,84 & 3,60 & 104 \\
\hline Acenaftileno & 6,26 & 0,20 & 6,21 & 1,35 & 99 \\
\hline Acenafteno & 5,55 & 0,13 & 5,50 & 0,97 & 99 \\
\hline Fluoreno & 4,71 & 0,11 & 4,60 & 2,36 & 98 \\
\hline Fenantreno & 11,57 & 0,12 & 11,01 & 4,86 & 95 \\
\hline Antraceno & 3,74 & 0,05 & 3,80 & 1,84 & 102 \\
\hline Fluoranteno & 8,32 & 0,09 & 8,36 & 0,39 & 100 \\
\hline Pireno & 8,95 & 0,08 & 8,92 & 2,37 & 100 \\
\hline Benzo(a)antraceno & 4,42 & 0,08 & 4,19 & 5,11 & 95 \\
\hline Criseno & 4,62 & 0,11 & 4,34 & 6,03 & 94 \\
\hline Benzo(b)fluoranteno & 7,86 & 0,10 & $(\mathrm{~A})$ & ----- & ----- \\
\hline Benzo(k)fluoranteno & 3,444 & 0,036 & $(\mathrm{~A})$ & ----- & ----- \\
\hline Benzo(a)pireno & 4,71 & 0,17 & 4,51 & 4,31 & 96 \\
\hline Indeno(1,2,3-cd)pireno & 4,425 & 0,030 & 4,06 & 8,34 & 92 \\
\hline Dibenzo(a,h)antraceno & 4,555 & 0,063 & (B) & ----- & ---- \\
\hline Benzo(ghi)perileno & 5,669 & 0,069 & 5,33 & 5,94 & 94 \\
\hline
\end{tabular}

$\mathrm{U}=$ Incerteza Expandida; $\mathrm{ER}$ = Erro Relativo ; Recup. = Recuperação;

(A) coelui com Benzo(j)fluoranteno; (B) coelui com Dibenzo(a,c)antraceno.

\section{DISCUSSÕES}

Os resultados obtidos para as replicatas foram tratados estatisticamente empregando o Teste de Grubbs, para a verificação dos valores aberrantes (ISO 5725, 1994).

Para qualquer método quantitativo, existe uma faixa de concentração do analito ou valores da propriedade no qual o método pode ser aplicado, sendo esta uma faixa que apresente condições de linearidade. No limite inferior da faixa de concentração, os fatores limitantes são os valores dos limites de detecção e de quantificação. No limite superior, os fatores limitantes dependem do sistema de resposta do equipamento de medição (INMETRO, 2007).

A linearidade foi determinada utilizando-se o método matemático conhecido como análise de regressão linear. Foram determinados os coeficientes de regressão angular (a) e linear (b), bem como o coeficiente de correlação quadrado (r2). Este último parâmetro permite uma estimativa da qualidade da curva obtida, pois quanto mais próximo de 1,0, menor a dispersão do conjunto de pontos experimentais e menor a incerteza dos coeficientes de regressão estimados. As curvas de calibração para cada analito apresentaram coeficiente de correlação quadrado maior que 0,995, indicando um ajuste ideal dos dados para a linha de regressão. $O$ valor do coeficiente linear sempre próximo a zero indicou que o sistema está livre de tendências. O método apresentou-se linear para a faixa de trabalho utilizada e os resíduos da curva de calibração apresentaram distribuição aleatória. O método apresentou-se linear na faixa de trabalho (1 a $11 \mu \mathrm{g} / \mathrm{g}$ ).

O método dos mínimos quadrados supõe que os resíduos têm a mesma variância. Na calibração isto significa que a dispersão das medidas é independente do valor da concentração. Esta condição de variância uniforme é chamada 
Homocedasticidade. Para verificar se o sistema é homocedástico (variâncias iguais) ou heterocedástico (variâncias diferentes) foi aplicado o Teste de Cochran (ISO 5725, 1994), para avaliar a variância ao longo da curva. Conforme observado na Tabela 2, alguns HPAs apresentaram comportamento heterocedástico, ou seja, variâncias diferentes. Porém este fato não foi considerado crítico porque o maior desvio padrão relativo (DPR) encontrado nos sistemas heterocedásticos foi de 5,54 $\%$ (BkFluo), semelhante ao maior DPR encontrado para os sistemas homocedásticos, que foi de $6,78 \%$ (BaP).

O limite de detecção (LD) é a menor quantidade do analito presente em uma amostra que pode ser detectado e o limite de quantificação (LQ) é definido como a menor concentração do analito que pode ser quantificada na amostra com exatidão e precisão aceitáveis, sob as condições de análise utilizadas (EURACHEM, 1998).

O LD e o LQ foram determinados utilizando o desvio padrão (S) de 10 replicatas de branco com adição de pequena concentração dos analitos $(0,1 \mu \mathrm{g} / \mathrm{g})$, de acordo com as equações (1) e (2), respectivamente.

$$
\begin{gathered}
L D=3 * S \\
L Q=10 * S
\end{gathered}
$$

Os valores de LD e LQ encontrados para cada HPA estão descritos na Tabela 3. Os valores de LD encontrados estão entre $0,004 \mu \mathrm{g} / \mathrm{g}$ para o naftaleno e 0,051 $\mu \mathrm{g} / \mathrm{g}$ para o Benzo(k)fluoranteno. Os valores de $\mathrm{LQ}$ encontrados estão entre 0,013 $\mu \mathrm{g} / \mathrm{g}$ para o naftaleno e $0,171 \mu \mathrm{g} / \mathrm{g}$ para o Benzo(k)fluoranteno.

A precisão é um termo geral para avaliar a dispersão de resultados entre ensaios independentes, repetidos de uma mesma amostra, amostras semelhantes ou padrões, em condições definidas (INMETRO, 2007). A precisão do método foi determinada através de estudos de repetitividade, calculando-se o desvio padrão relativo (\% DPR), das amostras analisadas em replicata (Thompson et al. 2002, p. 196-204). Com esse intuito a equação 3 foi aplicada, onde s é o desvio padrão das leituras no nível de concentração estudado e ${ }^{\bar{y}}$ é a média dos resultados obtidos.

$$
R S D(\%)=\frac{s}{\bar{y}} * 100
$$

Na Tabela 3 estão descritos os valores mínimos e máximos de DPR(\%) encontrados na faixa de trabalho para cada HPA. Todos os valores encontrados estão abaixo de $5 \%$, sendo considerado aceitável para o método.

A exatidão de um método analítico é a concordância dos resultados obtidos pelo método em estudo em relação ao valor de referência aceito convencionalmente como verdadeiro (Thompson et al. 2002, p. 196-204; INMETRO, 2007). Os processos mais utilizados para avaliar a exatidão de um método são: materiais de referência; comparação de métodos, participação em ensaios interlaboratoriais e realização de ensaios de recuperação (INMETRO, 2007, Ribani et al. 2004, p. 771780).

A exatidão do método foi determinada através de ensaios de recuperação em toda a faixa de trabalho para cada HPA. Na Tabela 3 estão descritos os valores mínimos e máximos de recuperação (\%) encontrados para cada HPA. Todos os valores encontrados estão entre $94 \%$ e $103 \%$, sendo considerado aceitável para o método.

A exatidão também foi avaliada através do uso de material de referência certificado. O MRC 2260a fornecido pelo NIST possui 36 HPAs em tolueno (SRM 2260a, 2004), o que acarretou problemas de coeluição com o Benzo(b)fluoranteno 
Benzo(k)fluoranteno e Dibenzo(a,h)antraceno. Os outros treze HPAs foram quantificados e apresentaram recuperação de $92 \%$ a $104 \%$ e erro relativo (ER\%) de $0,39 \%$ a $8,34 \%$, conforme apresentado na Tabela 4 . Estes resultados são compatíveis com os dados obtidos nos ensaios de recuperação com adição padrão, descritos na Tabela 3.

\title{
6 CONCLUSÕES
}

O método CG/EMDI foi otimizado e validado para a determinação de HPAs em solução. Todos os parâmetros de desempenho avaliados foram adequados ao escopo do estudo. O método desenvolvido apresentou boa exatidão e precisão, indicando a eficiência do método de quantificação utilizado.

O uso da espectrometria de massas com diluição isotópica (EMDI), ou seja, um método primário de análise, confere rastreabilidade e confiabilidade aos resultados.

Estes resultados são importantes subsídios para o trabalho que o Inmetro está desenvolvendo no sentido de produzir e certificar materiais de referência para HPAs.

\begin{abstract}
This work had as aim the validatation of an analytical method for determination of sixteen polycyclic aromatic hydrocarbons (PAHs) in solution. The technique of CG/MS combined with isotopic dilution principle was used for better accuracy and precision of the results. The performance parameters of the method had been determined and joined results had been satisfactory.
\end{abstract}

Keywords: polycyclic aromatic hydrocarbons (PAHs), validation, Certified reference material (CRM)

\section{Agradecimentos}

Os autores agradecem ao apoio financeiro do CNPq/PROMETRO (554479/2006-0) e FINEP. 


\section{REFERÊNCIAS}

AOAC. Guidelines for Single Laboratory Validation of Analytical Methods for Tracelevel Concentrations of Organic Chemicals, Washington, 1999.

DE BIÈVRE, P.; TAYLOR, P. D. P. Traceability to the SI of amount-of-substance measurement: from ignoring to realizing, a chemist's view. Metrologia, v. 34, p. 6775, 1997.

EURACHEM Guide. The Fitness for Purpose of Analytical Methods: A Laboratory Guide to Method Validation and Related Topics, v. 61, 1998.

IARC. Mixtures and Exposures Evaluated and Their Classification. International Agency for Research on Cancer, Lyon, France, 2007.

INMETRO, Orientação sobre validação de métodos de ensaios químicos, DOQCGCRE-008, 2007.

ISO $5725(\mathrm{E})$. Accuracy (trueness and precision) of measurement methods and results, 1994.

ISO/IEC 17025:2005. General requirements for the competence of calibration and testing laboratories, International Organization for Standardization, Switzerland, Geneva, 2005.

ITOH, N.; NUMATA, M.; AOYAGI, Y.; YARITA, T. Effect of residues remaining in the injection liner of a gas chromatograph on the quantification of polycyclic aromatic hydrocarbons by isotope dilution mass spectrometry using deuterium-labeled internal standards. Journal of Chromatography A, v. 1134, n. 1-2, p. 246-252, 2006.

MASTRAL, M.; CALLÉN, M. A Review on Polycyclic Aromatic Hydrocarbon (PAH) Emissions from Energy Generation. Environmental Science \& Technology, v. 34, n. 15, p. 3051-3057, Jul. 2000.

MILTON, M.; QUINN, T. Primary methods for the measurement of amount of substance. Metrologia, v. 38, p. 289-296, 2001.

PLANAS, C.; PUIG, A.; RIVERA, J.; CAIXACH, J. Analysis of alkyl and 2-6-ringed polycyclic aromatic hydrocarbons by isotope dillution gas chromatography/mass spectrometry quality assurance and determination in Spanish river sediments. Journal of Chromatography A, n. 1-2, v. 1113, p. 220-230, 2006.

QUINN, T. Primary methods of measurement and primary standards. Metrologia, v. 34, p. 61-65, 1997. 
RIBANI, M.; BOTTOLI, C.; COLLINS, C.; JARDIM, I.; MELO, L. Validação em Métodos Cromatográficos e Eletroforéticos. Química Nova, v. 27, n. 5, p. 771-780, 2004.

SRM 2260a. Certificate of Analysis - Standard Reference Material - Aromatic Hydrocarbons in toluene, National Institute of Standards and Technology, Gaithersburg, USA, 2004.

THOMPSON, S; BUDZINSKI, H; LEMENACH, K; LETELLIER, M; GARRIGUES, P. Multi-residue analysis of polycyclic aromatic hydrocarbons, polychlorobiphenyls, and organochlorine pesticides in marine sediments. Anal Bioanal Chem, v. 372, n. 1, p. 196-204, 2002.

VEYRAND, B. ; BROSSEAUD, A. ; SARCHER, L. ; VARLET, V. ; MONTEAU, F. ; MARCHAND, P. ; ANDRE, F. ; BIZEC, B. Innovative method for determination of 19 polycyclic aromatic hydrocarbons in food and oil samples using gas chromatography coupled to tandem mass spectrometry based on an isotope dilution approach. Journal of Chromatography A, v. 11, n. 2, p. 333-344, 2007. 